Pacific Journal of Mathematics

CONNECTEDNESS TM KLEINEN AND LOCAL
CONNECTEDNESS IN $2^{X}$ AND $C(X)$ 


\section{CONNECTEDNESS IM KLEINEN AND LOCAL CONNECTEDNESS IN $2^{X}$ AND $C(X)$}

JACK T. Goodykoontz, JR.

Let $X$ be a compact connected metric space and $2^{X}(C(X))$ denote the hyperspace of closed subsets (subcontinua) of $X$. In this paper the hyperspaces are investigated with respect to point-wise connectivity properties. Let $M \in C(X)$. Then $2^{X}$ is locally connected (connected im kleinen) at $M$ if and only if for each open set $U$ containing $M$ there is a connected open set $V$ such that $M \subset V \subset U$ (there is a component of $U$ which contains $M$ in its interior). This theorem is used to prove the following main result. Let $A \in 2^{x}$. Then $2^{x}$ is locally connected (connected im kleinen) at $A$ if and only if $2^{x}$ is locally connected (connected im kleinen) at each component of $A$. Several related results about $C(X)$ are also obtained.

A continuum $X$ will be a compact connected metric space. $2^{X}(C(X))$ denotes the hyperspace of closed subsets (subcontinua) of $X$, each with the finite (Vietoris) topology, and since $X$ is a continuum, each of $2^{X}$ and $C(X)$ is also a continuum (see [5]).

One of the earliest results about hyperspaces of continua, due to Wojdyslawski [7], was that each of $2^{X}$ and $C(X)$ is locally connected if and only if $X$ is locally connected. As a point-wise property, local connectedness is stronger than connectedness im kleinen, which in turn is stronger than aposyndesis. The author [1] has shown that if $X$ is any continuum, then each of $2^{X}$ and $C(X)$ is aposyndetic. It is the purpose of this paper to investigate the internal structure of $2^{X}$ and $C(X)$ with respect to these properties. In particular, we determine necessary and sufficient conditions (in terms of the neighborhood structure in $X$ ) that $2^{X}$ be locally connected at a point and that $2^{X}$ be connected im kleinen at a point. We also determine that $C(X)$ has, in general, stronger point-wise connectivity properties that either $2^{X}$ or $X$.

For notational purposes, small letters will denote elements of $X$, capital letters will denote subsets of $X$ and elements of $2^{X}$, and script letters will denote subsets of $2^{X}$. If $A \subset X$, then $A^{*}$ (int $A$ ) (bd $A$ ) will denote the closure (interior) (boundary) of $A$ in $X$.

Let $x \in X$. Then $X$ is locally connected (l.c.) at $x$ if for each open set $U$ containing $x$ there is a connected open set $V$ such that $x \in V \subset U . \quad X$ is connected im kleinen (c.i.k.) at $x$ if for each open set $U$ containing $x$ there is a component of $U$ which contains $x$ in its interior. $X$ is aposyndetic at $x$ if for each $y \in X-x$ there is a 
continuum $M$ such that $x \in \operatorname{int} M$ and $y \in X-M$.

If $A_{1}, \cdots, A_{n}$ are subsets of $X$, then $N\left(A_{1}, \cdots, A_{n}\right)=\left\{B \in 2^{X} \mid\right.$ for each $i=1, \cdots, n, B \cap A_{i} \neq \varnothing$, and $\left.B \subset \bigcup_{i=1}^{n} A_{\imath}\right\}$. The collection of all sets of the form $N\left(U_{1}, \cdots, U_{n}\right)$, with $U_{1}, \cdots, U_{n}$ open in $X$, is a base for the finite topology. It is easy to establish that

$$
N\left(U_{1}, \cdots, U_{n}\right)^{*}=N\left(U_{1}^{*}, \cdots, U_{n}^{*}\right)
$$

and that $N\left(V_{1}, \cdots, V_{m}\right) \subset N\left(U_{1}, \cdots, U_{n}\right)$ if and only if $\bigcup_{j=1}^{m} V_{\jmath} \subset$ $\bigcup_{i=1}^{n} U_{\imath}$ and for each $U_{\imath}$ there exists a $V_{j}$ such that $V_{j} \subset U_{i}$ (see [5]). We remark also that the finite topology is equivalent to the Hausdorff metric topology on $2^{X}$ whenever $X$ is a compact metric space (theorem on page 47 of [4]).

If $\mathscr{A} \subset 2^{X}$, then $\mathrm{U}\{A \mid A \in \mathscr{A}\}$ is open (closed) in $X$ whenever $\mathscr{A}$ is open (closed) in $2^{X}$ (see [5]). Furthermore, if $\mathscr{A} \cap C(X) \neq \varnothing$ and $\mathscr{A}$ is connected, then $\bigcup\{A \mid A \in \mathscr{A}\}$ is connected (Lemma 1.2 of [3]).

If $n$ is a positive integer, then $F_{n}(X)=\left\{A \in 2^{X} \mid A\right.$ has at most $n$ elements $\}$ and $F(X)=\bigcup_{n=1}^{\infty} F_{n}(X)$.

An order arc in $2^{X}(C(X))$ is an arc which is also a chain with respect to the partial order on $2^{X}(C(X))$ induced by set inclusion. If $A, B \in 2^{x}$, then there exists an order arc from $A$ to $B$ if and only if $A \subset B$ and each component of $B$ meets $A$ (Lemma 2.3 of [3]). It follows (Lemma 2.6 of [3]) that every order arc whose initial point is an element of $C(X)$ is entirely contained within $C(X)$.

It will be convenient to begin our study by considering points of $C(X)$.

THEOREM 1. Let $M \in C(X)$. Then $2^{X}$ is c.i.k. at $M$ if and only if for each open set $U$ containing $M$ there is a component of $U$ which contains $M$ in its interior.

Proof. Suppose $2^{X}$ is c.i.k. at $M$. Let $U$ be an open set containing $M$. Then $M \in N(U)$, so there exists a component $\mathscr{C}$ of $N(U)$ containing $M$ in its interior. It follows that $\bigcup\{A \mid A \in \mathscr{C}\}$ is a connected set containing $M$ in its interior and lying in $U$.

Conversely, suppose that for each open set $U$ containing $M$ there is a component of $U$ which contains $M$ in its interior. Let $N\left(U_{1}, \cdots, U_{n}\right)$ be a basic open set containing $M$ and let $N\left(V_{1}, \cdots, V_{m}\right)$ be a basic open set such that $M \in N\left(V_{1}, \cdots, V_{m}\right) \subset N\left(V_{1}, \cdots, V_{m}\right)^{*} \subset$ $N\left(U_{1}, \cdots, U_{n}\right)$. Let $V=\bigcup_{i=1}^{m} V_{i}$. Then there is a component $C$ of $V$ which contains $M$ in its interior. For each $i=1, \cdots, m$, let $W_{i}=V_{\imath} \cap$ int $C$. Then $M \in N\left(W_{1}, \cdots, W_{m}\right) \subset N\left(V_{1}, \cdots, V_{m}\right)$. If $A \in$ $N\left(W_{1}, \cdots, W_{m}\right)$, then $A \subset C^{*}$, and $A, \quad C^{*} \in N\left(V_{1}^{*}, \cdots, V_{m}^{*}\right)=$ 
$N\left(V_{1}, \cdots, V_{m}\right)^{*} \subset N\left(U_{1}, \cdots, U_{n}\right)$. Since $C^{*}$ is connected there exists an order arc in $N\left(U_{1}, \cdots, U_{n}\right)$ from $A$ to $C^{*}$. It follows that there is a component of $N\left(U_{1}, \cdots, U_{n}\right)$ which contains $M$ in its interior.

Corollary 1. Let $x \in X$. Then $2^{X}$ is c.i.k. at $\{x\}$ if and only if $X$ is c.i.k. at $x$.

Lemma 1. Let $V$ be a connected open set and $V_{1}, \cdots, V_{n}$ be open sets such that $\bigcup_{i=1}^{n} V_{i}=V$. Then $N\left(V_{1}, \cdots, V_{n}\right)$ is connected.

Proof. Let $p$ be the smallest positive integer such that $F_{p}(X)_{i}^{\prime} \cap$ $N\left(V_{1}, \cdots, V_{n}\right) \neq \varnothing$. We will show that

$$
\mathscr{F}=\bigcup_{i=p}^{\infty}\left(F_{i}(X) \cap N\left(V_{1}, \cdots, V_{n}\right)\right)
$$

is connected.

Let $\mathscr{A}=\left\{\left\{x_{1}, \cdots, x_{n}\right\} \mid\right.$ for each $i=1, \cdots, n, x_{i} \in V_{i}$, and $x_{i}=x_{j}$ if and only if $i=j\}$. We will first establish that $\mathscr{A}$ lies in a connected subset of $\mathscr{F}$. Let $\left\{x_{1}, \cdots, x_{n}\right\},\left\{y_{1}, \cdots, y_{n}\right\} \in \mathscr{A}$. Define $\mathscr{A}_{1}=$ $\left\{\left\{x_{1}, \cdots, x_{n}, y\right\} \mid y \in V\right\}$ and $\mathscr{B}_{1}=\left\{\left\{y_{1}, x_{2}, \cdots, x_{n}, y\right\} \mid y \in V\right\}$. Then each of $\mathscr{A}_{1}$ and $\mathscr{B}_{1}$ is the continuous image of the connected set $V$, so $\mathscr{A}_{1}$ is a connected subset of $\mathscr{F}$ which contains $\left\{x_{1}, \cdots, x_{n}\right\}$ and $\left\{x_{1}, \cdots, x_{n}, y_{1}\right\}$ and $\mathscr{B}_{1}$ is a connected subset of $\mathscr{F}$ which contains $\left\{x_{1}, \cdots, x_{n}, y_{1}\right\}$ and $\left\{y_{1}, x_{2}, \cdots, x_{n}\right\}$. Similarly, for each $i=2, \cdots, n-1$ define $\mathscr{A}_{i}=\left\{\left\{y_{1}, \cdots, y_{i-1}, x_{i}, \cdots, x_{n}, y\right\} \mid y \in V\right\}$ and

$$
\mathscr{B}_{i}=\left\{\left\{y_{1}, \cdots, y_{i}, x_{i+1}, \cdots, x_{n}, y\right\} \mid y \in V\right\} \text {. }
$$

Then $\mathscr{A}_{i}$ is a connected subset of $\mathscr{F}$ which contains $\left\{y_{1}, \cdots, y_{i-1}\right.$, $\left.x_{i}, \cdots, x_{n}\right\}$ and $\left\{y_{1}, \cdots, y_{i}, x_{i}, \cdots, x_{n}\right\}$ and $\mathscr{B}_{i}$ is a connected subset of $\mathscr{F}$ which contains $\left\{y_{1}, \cdots, y_{i}, x_{i}, \cdots, x_{n}\right\}$ and $\left\{y_{1}, \cdots, y_{i}, x_{i+1}, \cdots, x_{n}\right\}$. Define $\mathscr{A}_{n}=\left\{\left\{y_{1}, \cdots, y_{n-1}, x_{n}, y\right\} \mid y \in V\right\}$ and

$$
\mathscr{B}_{n}=\left\{\left\{y_{1}, \cdots, y_{n}, y\right\} \mid y \in V\right\} \text {. }
$$

Then $\mathscr{A}_{n}$ is a connected subset of $\mathscr{F}$ which contains $\left\{y_{1}, \cdots, y_{n-1}, x_{n}\right\}$ and $\left\{y_{1}, \cdots, y_{n}, x_{n}\right\}$ and $\mathscr{B}_{n}$ is a connected subset of $\mathscr{F}$ which contains $\left\{y_{1}, \cdots, y_{n}, x_{n}\right\}$ and $\left\{y_{1}, \cdots, y_{n}\right\}$. It follows that $\bigcup_{i=1}^{n}\left(\mathscr{A}_{i} \cup \mathscr{B}_{i}\right)$ is a connected subset of $\mathscr{F}$ which contains $\left\{x_{1}, \cdots, x_{n}\right\}$ and $\left\{y_{1}, \cdots, y_{n}\right\}$.

Now let $\left\{x_{1}, \cdots, x_{m}\right\} \in \mathscr{F}-\mathscr{A}$. If $p \leqq m<n$, choose $n-m$ distinct elements $x_{m+1}, \cdots, x_{n}$ such that $\left\{x_{1}, \cdots, x_{m}, x_{m+1}, \cdots, x_{n}\right\} \in \mathscr{A}$. For each $i=1, \cdots, n-m$ let $\mathscr{C}_{i}=\left\{\left\{x_{1}, \cdots, x_{m+(i-1)}, y\right\} \mid y \in V\right\}$. Then $\mathscr{C}_{i}$ is a connected subset of $\mathscr{F}$ containing $\left\{x_{1}, \cdots, x_{m+(i-1)}\right\}$ and $\left\{x_{1}, \cdots, x_{m+i}\right\}$. Hence $\bigcup_{i=1}^{n-m} \mathscr{C}_{i}$ is a connected subset of $\mathscr{F}$ containing $\left\{x_{1}, \cdots, x_{m}\right\}$ and $\left\{x_{1}, \cdots, x_{n}\right\}$.

If $m \geqq n$, let $\left\{y_{1}, \cdots, y_{n}\right\} \in \mathscr{A}$. Let $\mathscr{D}_{1}=\left\{\left\{x_{1}, \cdots, x_{m}, y\right\} \mid y \in V\right\}$. 
Then $\mathscr{D}_{1}$ is a connected subset of $\mathscr{F}$ containing $\left\{x_{1}, \cdots, x_{m}\right\}$ and $\left\{x_{1}, \cdots, x_{m}, y_{1}\right\}$. For each $i=2, \cdots, n$, let $\mathscr{D}_{i}=\left\{\left\{x_{1}, \cdots, x_{m}, y_{1}, \cdots\right.\right.$, $\left.\left.y_{i-1}, y\right\} \mid y \in V\right\}$. Then $\mathscr{D}_{i}$ is a connnected subset of $\mathscr{F}$ containing $\left\{x_{1}, \cdots, x_{m}, y_{1}, \cdots, y_{i-1}\right\}$ and $\left\{x_{1}, \cdots, x_{m}, y_{1}, \cdots, y_{i}\right\}$. Hence $\bigcup_{i=1}^{n} \mathscr{D}_{2}$ is a connected subset of $\mathscr{F}$ containing $\left\{x_{1}, \cdots, x_{m}\right\}$ and $\left\{x_{1}, \cdots, x_{m}\right.$, $\left.y_{1}, \cdots, y_{n}\right\}$. With an analogous construction we can show that there is a connected subset of $\mathscr{F}$ which contains $\left\{y_{1}, \cdots, y_{n}\right\}$ and $\left\{x_{1}, \cdots\right.$, $\left.x_{m}, y_{1}, \cdots, y_{n}\right\}$. It follows that there is a connected subset of $\mathscr{F}$ which contains $\left\{x_{1}, \cdots, x_{m}\right\}$ and $\left\{y_{1}, \cdots, y_{n}\right\}$.

We have now established that $\mathscr{A}$ lies in a connected subset of $\mathscr{F}$ and that each member of $\mathscr{F}-\mathscr{A}$ lies in a connected subset of $\mathscr{F}$ which meets $\mathscr{A}$. Hence $\mathscr{F}$ is connected. Since $\mathscr{F}$ is dense in $N\left(V_{1}, \cdots, V_{n}\right)$, it follows that $N\left(V_{1}, \cdots, V_{n}\right)$ is connected.

Theorem 2. Let $M \in C(X)$. Then $2^{X}$ is l.c. at $M$ if and only if for each open set $U$ containing $M$ there exists a connected open set $V$ such that $M \subset V \subset U$.

Proof. Suppose $2^{X}$ is l.c. at $M$. Let $U$ be an open set containing $M$. Then $M \in N(U)$, so there exists a connected open set $\mathscr{V}$ such that $M \in \mathscr{V} \subset N(U)$. It follows that $M \subset \bigcup\{A \mid A \in \mathscr{V}\}=V \subset U$, and $V$ is open and connected.

Conversely, suppose that for each open set $U$ containing $M$ there exists a connected open set $V$ such that $M \subset V \subset U$. Let $N\left(U_{1}, \cdots\right.$, $\left.U_{n}\right)$ be a basic open set such that $M \in N\left(U_{1}, \cdots, U_{n}\right)$ and let $U=$ $\bigcup_{i=1}^{n} U_{i}$. Then there exists a connected open set $V$ such that $M \subset$ $V \subset U$. Let $V_{i}=V \cap U_{\imath}$. Then $M \in N\left(V_{1}, \cdots, V_{n}\right) \subset N\left(U_{1}, \cdots, U_{n}\right)$, and by Lemma $1, N\left(V_{1}, \cdots, V_{n}\right)$ is connected.

Corollary 2. Let $x \in X$. Then $2^{X}$ is l.c. at $\{x\}$ if and only if $X$ is l.c. at $x$.

We remark that if $M \in C(X)$ and $2^{X}$ is 1.c. at $M$, then Lemma 1 and Theorem 2 imply the existence of a local base of connected sets at $M$, each of which is of the form $N\left(U_{1}, \cdots, U_{n}\right)$.

The next several results concern the relationships between $2^{X}$ and $C(X)$ with respect to local connectedness and connectedness im kleinen at points of $C(X)$.

Theorem 3. Let $M \in C(X)$. If $2^{X}$ is c.i.k. at $M$, then $C(X)$ is c.i.k. at $M$.

Proof. Let $N\left(U_{1}, \cdots, U_{n}\right) \cap C(X)$ be an open set containing $M$. Let $N\left(V_{1}, \cdots, V_{m}\right)$ be an open set such that $M \in N\left(V_{1}, \cdots, V_{m}\right) \subset$ 
$N\left(V_{1}, \cdots, V_{m}\right)^{*} \subset N\left(U_{1}, \cdots, U_{n}\right)$. Since $2^{X}$ is c.i.k. at $M$, there exists an open set $N\left(W_{1}, \cdots, W_{k}\right)$ such that

$$
M \in N\left(W_{1}, \cdots, W_{k}\right) \subset N\left(V_{1}, \cdots, V_{m}\right)
$$

and with the property that $B \in N\left(W_{1}, \cdots, W_{k}\right)$ implies $N\left(V_{1}, \cdots, V_{m}\right)$ contains a connected set containing $B$ and $M$. Then $N\left(U_{1}, \cdots, U_{n}\right)$ contains a continuum containing $B$ and $M$.

Let $K \in N\left(W_{1}, \cdots, W_{k}\right) \cap C(X)$. Then there exists a continuum $\mathscr{L}$ in $N\left(U_{1}, \cdots, U_{n}\right)$ containing $K$ and $M$. Now $\bigcup\{A \mid A \in \mathscr{L}\}=$ $L \in C(X)$, and $L \in N\left(U_{1}, \cdots, U_{n}\right)$, since $\mathscr{L} \subset N\left(U_{1}, \cdots, U_{n}\right)$. It follows that there exist order arcs $\mathscr{L}_{K}$ and $\mathscr{L}_{M}$ in $N\left(U_{1}, \cdots, U_{n}\right) \cap$ $C(X)$ from $K$ to $L$ and from $M$ to $L$. So $\mathscr{L}_{K} \cup \mathscr{L}_{M}$ is a continuum in $N\left(U_{1}, \cdots, U_{n}\right) \cap C(X)$ containing $K$ and $M$. Hence $C(X)$ is c.i.k. at $M$.

CoROllary 3. Let $M \in C(X)$. If for each open set $U$ containing $M$ there is a component of $U$ which contains $M$ in its interior, then $C(X)$ is c.i.k. at $M$.

Corollary 3 is a generalization of Theorem 6 of [6]. The example following Theorem 6 of [6] shows that the converse of Corollary 3 is false. It also shows that the converse of Question 1 below is false.

Question 1. Let $M \in C(X)$. If $2^{X}$ is l.c. at $M$, is $C(X)$ l.c. at $M$ ?

Corollary 4. Let $x \in X$. Then $X$ is c.i.k. at $x$ if and only if $C(X)$ is c.i.k. at $\{x\}$.

Proof. If $X$ is c.i.k. at $x$, then by Corollary $1,2^{x}$ is c.i.k. at $\{x\}$, and by Theorem $3, C(X)$ is c.i.k. at $\{x\}$.

Suppose $C(X)$ is c.i.k. at $\{x\}$. Let $U$ be an open set containing $x$. Then $\{x\} \in N(U) \cap C(X)$, so there exists an open set $N(V) \cap C(X)$, $\{x\} \in N(V) \cap C(X) \subset N(U) \cap C(X)$, with the property that $M \in$ $N(V) \cap C(X)$ implies $N(U) \cap C(X)$ contains a connected set containing $M$ and $\{x\}$.

Now $x \in V \subset U$. Let $y \in V$. Then $\{y\} \in N(V) \cap C(X)$, so $N(U) \cap$ $C(X)$ contains a connected set $\mathscr{L}$ containing $\{y\}$ and $\{x\}$. It follows that $U\{L \mid L \in \mathscr{L}\}$ is a connected subset of $U$ containing $x$ and $y$. Hence $X$ is c.i.k. at $x$.

Corollary 5. Let $x \in X$. If $X$ is l.c. at $x$, then $C(X)$ is l.c. at $\{x\}$. 
Proof. This follows from the observation that if $V$ is connected, then $N(V) \cap C(X)$ is connected, since each point of $(N(V) \cap$ $C(X))-F_{1}(V)$ can be joined by an order arc in $N(V) \cap C(X)$ to a point of $F_{1}(V)$, and $F_{1}(V)$ is connected. false.

The next example shows that the converse of Corollary 5 is

ExAMPLe 1. This example is from page 113 of [2]. For each positive integer $n$ and each positive integer $m$ let $L_{n, m}$ denote the line segment in the plane from $\left(1 /(n+1),(-1)^{n+1} 1 / m(n+1)\right)$ to $(1 / n, 0)$. Let $A_{n}=\left(\bigcup_{m=1}^{\infty} L_{n, m}\right)^{*}$ and let $X=\left(\bigcup_{n=1}^{\infty} A_{n}\right)^{*}$. Then $X$ is c.i.k. at $(0,0)$ but is not l.c. at $(0,0)$.

We now give a brief argument that $C(X)$ is 1.c. at $\{(0,0)\}$. For each $n \geqq 2$ choose $q_{n}, r_{n}$, and $s_{n}$ so that $1 /(n+1)<q_{n}<r_{n}<1 / n<$ $s_{n}<1 /(n-1)$. Let $U_{n}=\left\{(x, y) \mid x<r_{n}\right\}$ and $V_{n}=\left\{(x, y) \mid q_{n}<x<s_{n}\right\}$. Then $N\left(U_{n}\right) \cup N\left(U_{n}, V_{n}\right)$ is a continuum-wise connected open set in $C(X)$ containing $\{(0,0)\}$, for if $M, N \in N\left(U_{n}\right) \cup N\left(U_{n}, V_{n}\right)$, then $M, N \subset\{(x, y) \mid x<1 / n\} \cup\left\{(x, 0) \mid 1 / n \leqq x<s_{n}\right\}$ and a continuum can be constructed in $C(X)$ containing $M$ and $N$ and lying in $N\left(U_{n}\right) \cup N\left(U_{n}, V_{n}\right)$. Clearly $\left\{N\left(U_{n}\right) \cup N\left(U_{n}, V_{n}\right) \mid n=2,3, \cdots\right\}$ is a neighborhood base at $\{(0,0)\}$.

The following definition and Lemma 2 concern the finite topology and will be used in proving our main results, in which we obtain necessary and sufficient conditions that $2^{X}$ be l.c. (c.i.k.) at an arbitrary point.

Let $A \in 2^{X}$. A basic open set $N\left(U_{1}, \cdots, U_{n}\right)$ is essential with respect to $A$ if $A \in N\left(U_{1}, \cdots, U_{n}\right)$ and for each $i=1, \cdots, n$, $A-\mathrm{U}_{j \neq i} U_{j} \neq \varnothing$.

Lemma 2. Let $A \in 2^{X}$ and $N\left(U_{1}, \cdots, U_{n}\right)$ be an open set containing $A$. Then there exists an open set $N\left(V_{1}, \cdots, V_{m}\right)$ such that $A \in N\left(V_{1}, \cdots, V_{m}\right) \subset N\left(U_{1}, \cdots, U_{n}\right)$ and $N\left(V_{1}, \cdots, V_{m}\right)$ is essential with respect to $A$.

Proof. Choose $x_{1}, \cdots, x_{n} \in A$ such that $x_{\imath} \in U_{i}$. Let $V_{1}, \cdots, V_{n}$ be open sets such that for each $i=1, \cdots, n, x_{i} \in V_{i} \subset \bigcap\left\{U_{j} \mid x_{i} \in U_{j}\right\}$ and with the additional property that $V_{i}=V_{j}$ if $x_{i}=x_{j}$ and $V_{i} \cap V_{j}=$ $\varnothing$ if $x_{i} \neq x_{j}$. Let $\left\{V_{1}, \cdots, V_{k}\right\}$ (relabeling if necessary) be the set of $V_{i}$ 's which are distinct. For each $y \in A-\bigcup_{i=1}^{k} V_{i}$ let $O_{y}$ be an open set such that $y \in O_{y} \subset \bigcap\left\{U_{j} \mid y \in U_{j}\right\}$ and such that $O_{y} \cap\left\{x_{1}, \cdots\right.$, $\left.x_{n}\right\}=\varnothing$. Since $A-\bigcup_{i=1}^{k} V_{i}$ is compact, there exist $y_{1}, \cdots, y_{p}$ such that $A-\bigcup_{i=1}^{k} V_{i} \subset \bigcup_{i=1}^{p} O_{y_{i}}$. We may assume that all the $O_{y_{i}}$ 's are distinct. Let $\left\{O_{y_{1}}, \cdots, O_{y_{q}}\right\}$ (relabeling if necessary) be the subset of 
$\left\{O_{y_{1}}, \cdots, O_{y_{p}}\right\}$ consisting of all the $O_{y_{i}}$ 's with the property that $\left(A-\bigcup_{i=1}^{k} V_{i}\right)-\bigcup_{j \neq i} O_{y_{j}} \neq \varnothing$.

For notational purposes, for each $j=1, \cdots, q$ let $O_{y_{i}}=V_{k+j}$ and let $k+q=m$. Then $A \in N\left(V_{1}, \cdots, V_{k}, V_{k+1}, \cdots, V_{m}\right)$. Clearly

$$
N\left(V_{1}, \cdots, V_{k}, V_{k+1}, \cdots, V_{m}\right) \subset N\left(U_{1}, \cdots, U_{n}\right) \text {. }
$$

For each $j=1, \cdots, k$ there exists $x_{i} \in A$ such that $x_{i} \in V_{j}$ and $x_{i} \notin\left(\bigcup_{p=1}^{m} V_{p}\right)-V_{j}$. For each $j=k+1, \cdots, m$,

$$
\left(A-\bigcup_{i=1}^{k} V_{i}\right)-\bigcup_{\substack{i=k+1 \\ i \neq j}}^{m} V_{i} \neq \varnothing \text {, }
$$

so there exists $a_{j} \in V_{j} \cap\left(A-\bigcup_{i=1}^{k} V_{i}\right)$ such that $a_{j} \notin \bigcup_{i \neq j} V_{i}$. It follows that $N\left(V_{1}, \cdots, V_{m}\right)$ is essential with respect to $A$.

Theorem 4. Let $A \in 2^{x}$. Then $2^{x}$ is c.i.k. at $A$ if and only if $2^{X}$ is c.i.k. at each component of $A$.

Proof. Suppose that $2^{x}$ is c.i.k. at $A$. Let $A_{1}$ be a component of $A$ and let $W$ be an open set containing $A_{1}$. Let $U$ be an open set such that $A_{1} \subset U \subset U^{*} \subset W$ and such that (bd $\left.U\right) \cap A=\varnothing$. Let $\left\{U_{1}, \cdots, U_{n}\right\}$ be a finite cover of $A-U$ by open sets such that for each $i=1, \cdots, n, \quad U \cap U_{i}=\varnothing$ and $A \cap U_{i} \neq \varnothing$. Then $A \in$ $N\left(U, U_{1}, \cdots, U_{n}\right)$.

Let $\mathscr{C}$ be a component of $N\left(U, U_{1}, \cdots, U_{n}\right)$ which contains $A$ in its interior. Define $f: \mathscr{C} \rightarrow N(U)$ by $f(B)=B \cap U$. If $N\left(V_{1}, \cdots, V_{p}\right) \subset$ $N(U)$, then $f^{-1}\left(N\left(V_{1}, \cdots, V_{p}\right)\right)=N\left(V_{1}, \cdots, V_{p}, U_{1}, \cdots, U_{n}\right) \cap \mathscr{C}$, so $f$ is continuous. Hence $f(\mathscr{C})$ is connected.

Let $N\left(V_{1}, \cdots, V_{q}\right)$ be an open set such that $A \in N\left(V_{1}, \cdots, V_{q}\right) \subset$ $\mathscr{C}$. Let $\left\{V_{1}, \cdots, V_{m}\right\}$ (relabeling if necessary) be the largest subset of $\left\{V_{1}, \cdots, V_{q}\right\}$ with the property that for each $j=1, \cdots, m, V_{j} \cap U \neq \varnothing$. Let $\left\{V_{1}, \cdots, V_{k}\right\}$ (relabeling if necessary) be the largest subset of $V_{1}, \cdots, V_{m}$ with the property that for each $j=1, \cdots, k, V_{j} \cap\left(\bigcup_{i=1}^{n} U_{i}\right)=$ $\varnothing$. For each $j=1, \cdots, k$, let $V_{j}^{1}=V_{j} \cap U$ and $V_{j}^{2}=V_{j} \cap\left(\bigcup_{i=1}^{n} U_{i}\right)$. Then

$$
\begin{aligned}
A & \in N\left(V_{1}^{1}, \cdots, V_{k}^{1}, V_{k+1}, \cdots, V_{m}, V_{1}^{2}, \cdots, V_{k}^{2}, V_{m+1}, \cdots, V_{q}\right) \\
& =\mathscr{V} \subset N\left(V_{1}, \cdots, V_{q}\right) \subset \mathscr{C} .
\end{aligned}
$$

Now if $B \in \mathscr{Y}$, then

$$
\begin{aligned}
f(B) & =B \cap U \\
& =B \cap\left[\left(\bigcup_{j=1}^{k} V_{j}^{1}\right) \cup\left(\bigcup_{j=k+1}^{m} V_{j}\right)\right] \in N\left(V_{1}^{1}, \cdots, V_{k}^{1}, V_{k+1}, \cdots, V_{m}\right) .
\end{aligned}
$$

Conversely, suppose $C \in N\left(V_{1}^{1}, \cdots, V_{k}^{1}, V_{k+1}, \cdots, V_{m}\right)$. For each $j=$ $1, \cdots, k$, let $x_{j} \in V_{j}^{2}$ and for each $j=m+1, \cdots, q$ let $x_{j} \in V_{j}$. Then 
$C \cup\left\{x_{1}, \cdots, x_{k}, x_{m+1}, \cdots, x_{q}\right\} \in \mathscr{Y}$ and $f\left(C \cup\left\{x_{1}, \cdots, x_{k}, x_{m+1}, \cdots, x_{q}\right\}\right)=$ $C \in f(\mathscr{Y})$. Hence $f(\mathscr{Y})=N\left(V_{1}^{1}, \cdots, V_{k}^{1}, V_{k+1}, \cdots, V_{m}\right)$. So $f(\mathscr{C})$ contains an open set containing $A \cap U$.

Let $C=\bigcup\{f(B) \mid B \in \mathscr{C}\}$. Then $C^{*} \subset U^{*} \subset W$. Let $C\left(A_{1}\right)$ be the component of $C^{*}$ which contains $A_{1}$. Let $N\left(V_{1}, \cdots, V_{m}, V_{m+1}, \cdots, V_{p}\right)$ be an open set such that $A \in N\left(V_{1}, \cdots, V_{m}, V_{m+1}, \cdots, V_{p}\right) \subset \subset$ and such that $\bigcup_{i=1}^{m} V_{i} \subset U$ and $\bigcup_{i=m+1}^{p} V_{i} \subset \bigcup_{i=1}^{n} U_{i}$. Let $\left\{V_{1}, \cdots, V_{k}\right\}$ (relabeling if necessary) be the largest subset of $\left\{V_{1}, \cdots, V_{m}\right\}$ with the property that for each $i=1, \cdots, k, V_{i}^{*} \cap C\left(A_{1}\right)=\varnothing$. (A slight modification of the following argument is necessary in the case that $\left\{V_{1}, \cdots, V_{k}\right\}=\varnothing$.) Let $O$ be an open set containing $C\left(A_{1}\right)$ such that $O \cap\left(\bigcup_{i=1}^{k} V_{i}^{*}\right)=\varnothing$ and such that $($ bd $O) \cap C^{*}=\varnothing$.

Let $x \in A_{1}$. Suppose $x \notin \operatorname{int} C\left(A_{1}\right)$. Let $O_{x}$ be an open set containing $x$ such that $O_{x} \subset O \cap\left(\cap\left\{V_{i} \mid x \in V_{i}\right\}\right)$. Let $y \in O_{x}$ such that $y \notin C\left(A_{1}\right)$ and let $C(y)$ be the component of $C^{*}$ which contains $y$. Since $(\operatorname{bd} O) \cap C^{*}=\varnothing, C(y) \subset O$. Let $O_{y}$ be an open set containing $C(y)$ such that $O_{y} \subset O, O_{y} \cap C\left(A_{1}\right)=\varnothing$, and such that $\left(\right.$ bd $\left.O_{y}\right) \cap C^{*}=\varnothing$.

Now $O_{y}, O-O_{y}^{*}$, and $X-O^{*}$ are disjoint open sets, and $C^{*} \subset O_{y} \cup\left(O-O_{y}^{*}\right) \cup\left(X-O^{*}\right)$. Consequently the sets $N\left(O_{y}\right), N\left(O-O_{y}^{*}\right)$, $N\left(X-O^{*}\right), N\left(O_{y}, O-O_{y}^{*}\right), N\left(O_{y}, X-O^{*}\right), N\left(O-O_{y}^{*}, X-O^{*}\right)$, and $N\left(O_{y}, O-O_{y}^{*}, X-O^{*}\right)$ are pairwise disjoint, and $f(\mathscr{C})^{*}$ is contained in the union of these sets.

For each $i=1, \cdots, k$, let $x_{i} \in V_{i}$. For each $i=k+1, \cdots, m$, $C\left(A_{1}\right) \cap V_{i}^{*} \neq \varnothing$, and since $O-O_{y}^{*}$ is an open set containing $C\left(A_{1}\right)$, there exists $x_{i} \in O-O_{y}^{*}$ such that $x_{i} \in V_{i}$. Then $\left\{x_{1}, \cdots, x_{m}\right\},\left\{x_{1}, \cdots\right.$, $\left.x_{m}, y\right\} \in N\left(V_{1}, \cdots, V_{m}\right) \subset f(\mathscr{C})$. Furthermore, $\left\{x_{1}, \cdots, x_{m}\right\} \in N\left(O-O_{y}^{*}\right.$, $\left.X-O^{*}\right)$ and $\left\{x_{1}, \cdots, x_{m}, y\right\} \in N\left(O_{y}, O-O_{y}^{*}, X-O^{*}\right)$. Hence $f(\mathscr{C})^{*}$ is not connected, so $f(\mathscr{C})$ is not connected, a contradiction. Thus the assumption that $x \notin \operatorname{int} C\left(A_{1}\right)$ was false. It follows that $C\left(A_{1}\right)$ is a connected subset of $C^{*}$ which contains $A_{1}$ in its interior. Hence, by Theorem $1,2^{X}$ is c.i.k. at $A_{1}$.

For the converse, suppose that $2^{X}$ is c.i.k. at each component of $A$. Let $\mathscr{U}$ be an open set containing $A$ and $N\left(U_{1}, \cdots, U_{n}\right)$ be a basic open set such that $A \in N\left(U_{1}, \cdots, U_{n}\right) \subset N\left(U_{1}, \cdots, U_{n}\right) * \subset \mathscr{U}$. By Lemma 2 we may assume that $N\left(U_{1}, \cdots, U_{n}\right)$ is essential with respect to $A$. For each component $A_{\alpha}$ of $A$ let $\left\{U_{i_{1}}, \cdots, U_{i_{n_{\alpha}}}\right\}$ be the largest subset of $\left\{U_{1}, \cdots, U_{n}\right\}$ with the property that for $j=$ $1, \cdots, n_{\alpha}, \quad A_{\alpha} \cap U_{i_{j}} \neq \varnothing$. Then $A_{\alpha} \in N\left(U_{i_{1}}, \cdots, U_{i_{n_{\alpha}}}\right)$. Let $U_{\alpha}=$ $\bigcup_{j=1}^{n}{ }_{j} U_{i_{j}}$. By Theorem 1 there is a component $M_{\alpha}$ of $U_{\alpha}$ which contains $A_{\alpha}$ in its interior. For each $j=1, \cdots, n_{\alpha}$ let $V_{j}^{\alpha}=\left(\right.$ int $\left.M_{\alpha}\right) \cap$ $U_{i_{j}}$. Then $A_{\alpha} \in N\left(V_{1}^{\alpha}, \cdots, V_{n_{\alpha}}^{\alpha}\right) \subset N\left(U_{i_{1}}, \cdots, U_{i_{n_{\alpha}}}\right)$.

Now $A \subset \bigcup_{\alpha}\left(\bigcup_{j}^{n_{1}{ }_{1}} V_{j}^{\alpha}\right)$ and since $A$ is compact there exists a finite subcover of $A$ of the form $\bigcup_{i=1}^{m}\left(\bigcup_{j=1}^{n \alpha_{i}} V_{j}^{\alpha_{i}}\right)$. Then 
$A \in N\left(V_{1}^{\alpha_{1}}, \cdots, V_{n_{\alpha_{1}}}^{\alpha_{1}}, \cdots, V_{1}^{\alpha_{m}}, \cdots, V_{n_{\alpha_{m}}}^{\alpha_{m}}\right) \subset N\left(U_{1}, \cdots, U_{n}\right)$.

The last inclusion follows from the construction and the fact that $N\left(U_{1}, \cdots, U_{n}\right)$ is essential with respect to $A$. Let $M=\bigcup_{i=1}^{m} M_{\alpha_{i}}^{*}$. Then $M \in N\left(U_{1}, \cdots, U_{n}\right)^{*}$.

Let $B \in N\left(V_{1}^{\alpha_{1}}, \cdots, V_{n_{\alpha_{1}}}^{\alpha_{1}}, \cdots, V_{1}^{\alpha_{m}}, \cdots, V_{n_{\alpha_{m}}}^{\alpha_{m}}\right)$. Note that $B=$ $\bigcup_{i=1}^{m}\left(B \cap \bigcup_{j=1}^{n_{\alpha_{j}}} V_{j}^{\alpha_{i} *}\right)$. Now $B \cap\left(\bigcup_{j=1}^{n_{\alpha_{1}}} V_{j}^{\alpha_{1} *}\right) \subset M_{\alpha_{1}}^{*}$, so there exists an order arc $\mathscr{B}_{\alpha_{1}}$ from $B \cap\left(\bigcup_{j=1}^{n_{\alpha_{1}}} V_{j}^{\alpha_{1} *}\right)$ to $M_{\alpha_{1}}^{*}$. Define $f_{1}: \mathscr{B}_{\alpha_{1}} \rightarrow \mathscr{U}$ by $f_{1}(C)=$ $C \cup\left(\bigcup_{i=2}^{m}\left(B \cap \bigcup_{j=1}^{n_{\alpha_{i}}} V_{j}^{\alpha_{i}{ }^{*}}\right)\right)$. Since union is continuous, $f_{1}\left(\mathscr{B}_{\alpha_{1}}\right)$ is connected, and $B, M_{a_{1}}^{*} \cup\left(\bigcup_{i=2}^{m}\left(B \cap \bigcup_{j=1}^{n_{\alpha_{i}}} V_{j}^{\alpha_{i} *}\right)\right) \in f_{1}\left(\mathscr{B}_{\alpha_{1}}\right)$. For each $i=$ $2, \cdots, m$, there exists an order arc $\mathscr{B}_{\alpha_{i}}$ from $B \cap\left(\bigcup_{j=1}^{n_{\alpha_{i}}} V_{j}^{\alpha_{i} *}\right)$ to $M_{\alpha_{i}}^{\alpha}$. For each $i=2, \cdots, m-1$, define $f_{i}\left(\mathscr{B}_{\alpha_{i}}\right) \rightarrow \mathscr{C}$ by

$$
f_{i}(C)=\left(\bigcup_{k=1}^{i-1} M_{\alpha_{k}}^{*}\right) \cup C \cup\left(\bigcup_{k=i+1}^{m}\left(B \cap \bigcup_{j=1}^{n_{\alpha_{k}}} V_{j}^{\alpha_{k} *}\right)\right) .
$$

Then $f_{i}\left(\mathscr{B}_{\alpha_{i}}\right)$ is a connected subset of $\mathscr{C}$ containing $\left(\bigcup_{k=1}^{i-1} M_{a_{k}}^{*}\right) \cup$ $\left(\bigcup_{k=i}^{m}\left(B \cap \bigcup_{j=1}^{n_{\alpha_{k}}} V_{j}^{a} k^{*}\right)\right)$ and $\left(\bigcup_{k=1}^{i} M_{\alpha_{k}}^{*}\right) \cup\left(\bigcup_{k=i+1}^{m}\left(B \cap \bigcup_{j=1}^{n_{\alpha_{k}}} V_{j}^{\alpha_{k} *}\right)\right)$. Define $f_{m}\left(\mathscr{B}_{\alpha_{m}}\right) \rightarrow \mathscr{U}$ by $f_{m}(C)=\left(\bigcup_{k=1}^{m-1} M_{\alpha_{k}}^{*}\right) \cup C$. Then $f_{m}\left(\mathscr{B}_{\alpha_{m}}\right)$ is a connected subset of $\mathscr{U}$ containing $\left(\bigcup_{k=1}^{m-1} M_{\alpha_{k}}^{*}\right) \cup\left(B \cap \bigcup_{j=1}^{n_{\alpha_{m}}} V_{j}^{\alpha_{m} *}\right)$ and $M$. Hence $\bigcup_{i=1}^{m} f_{i}\left(\mathscr{B}_{\alpha_{i}}\right)$ is a connected subset of $\mathscr{U}$ containing $B$ and $M$. It follows that $2^{X}$ is c.i.k. at $A$.

TheOREM 5. Let $A \in 2^{X}$. Then $2^{x}$ is l.c. at $A$ if and only if $2^{X}$ is l.c. at each component of $A$.

Proof. Suppose that $2^{X}$ is l.c. at $A$. Let $A_{1}$ be a component of $A$ and let $W$ be an open set containing $A_{1}$. Let $U$ be an open set such that $A_{1} \subset U \subset W$ aud such that $($ bd $U) \cap A=\varnothing$. Let $\left\{U_{1}, \cdots\right.$, $U_{n}$ \} be a finite cover of $A-U$ by open sets such that for each $i=1, \cdots, n, U \cap U_{i}=\varnothing$ and $A \cap U_{i} \neq \varnothing$. Then $A \in N\left(U, U_{1}, \cdots\right.$, $\left.U_{n}\right)$.

Let $\mathscr{V}$ be a connected open set such that $A \in \mathscr{V} \subset N\left(U, U_{1}, \cdots\right.$, $U_{n}$ ). Define $f: \mathscr{V} \rightarrow N(U)$ by $f(B)=B \cap U$. An argument similar to the one used in Theorem 5 will establish that $f$ is both continuous and open. Hence $f(\mathscr{V})$ is connected and open.

Let $V=\bigcup\{f(B) \mid B \in \mathscr{C}\}$. Then $V \subset U$. Let $Q\left(A_{1}\right)$ be the quasicomponent of $V$ which contains $A_{1}$ and let $x \in Q\left(A_{1}\right)$. Let $B \in \mathscr{V}$ such that $x \in f(B)$. Then there exists an open set $N\left(V_{1}, \cdots, V_{m}\right.$, $\left.V_{m+1}, \cdots, V_{p}\right)$ such that $B \in N\left(V_{1}, \cdots, V_{m}, V_{m+1}, \cdots, V_{p}\right) \subset N\left(V_{1}^{*}, \cdots\right.$, $\left.V_{m}^{*}, V_{m+1}, \cdots, V_{p}\right) \subset \mathscr{Y}$ and such that $\bigcup_{i=1}^{m} V_{\imath}^{*} \subset U$ and $\bigcup_{i=m+1}^{p} V_{i} \subset$ $\bigcup_{i=1}^{n} U_{i}$. Let $\left\{V_{i}, \cdots, V_{k}\right\}$ be the largest subset of $\left\{V_{1}, \cdots, V_{m}\right\}$ with the property that for each $i=1, \cdots, k, V_{i}^{*} \cap Q\left(A_{1}\right)=\varnothing$. (A slight modification of the following argument is necessary in the case that 
$\left.\left\{V_{1}, \cdots, V_{k}\right\}=\varnothing.\right)$ Since $\bigcup_{i=1}^{k} V_{i}^{*}$ is compact, there exist disjoint open-closed sets $S$ and $T$ such that $\bigcup_{i=1}^{k} V_{i}^{*} \subset S, Q\left(A_{1}\right) \subset T$ and $S \cup T=V$.

Suppose $x \notin \operatorname{int} Q\left(A_{1}\right)$. Let $O$ be an open set containing $x$ such that $O \subset T \cap\left(\cap\left\{V_{\imath} \mid x \in V_{i}\right\}\right)$. Let $y \in O$ such that $y \notin Q\left(A_{1}\right)$. Then there exist disjoint open-closed sets $T^{\prime}$ and $T^{\prime \prime}$ such that $Q\left(A_{1}\right) \subset$ $T^{\prime}, y \in T^{\prime \prime}$, and $T^{\prime} \cup T^{\prime \prime}=T$.

Now $T^{\prime}, T^{\prime \prime}$, and $S$ are disjoint open sets whose union is $V$. Consequently the sets $N\left(T^{\prime}\right), \quad N\left(T^{\prime \prime}\right), \quad N(S), \quad N\left(T^{\prime}, T^{\prime \prime}\right), \quad N\left(T^{\prime}, S\right)$, $N\left(T^{\prime \prime}, S\right)$, and $N\left(T^{\prime}, T^{\prime \prime}, S\right)$ are pairwise disjoint and $f(\mathscr{Y})$ is contained in the union of these sets.

For each $i=1, \cdots, k$, let $x_{i} \in V_{i}$. For each $i=k+1, \cdots, m$, $Q\left(A_{1}\right) \cap V_{i}^{*} \neq \varnothing$, and since $T^{\prime}$ is an open set containing $Q\left(A_{1}\right)$, there exists $x_{i} \in T^{\prime}$ such that $x_{i} \in V_{i}$. Then

$$
\left\{x_{1}, \cdots, x_{m}\right\},\left\{x_{1}, \cdots, x_{m}, y\right\} \in N\left(V_{1}, \cdots, V_{m}\right) \subset f(\mathscr{Y}) .
$$

Furthermore, $\left\{x_{1}, \cdots, x_{m}\right\} \in N\left(T^{\prime}, S\right)$ and $\left\{x_{1}, \cdots, x_{m}, y\right\} \in N\left(T^{\prime}, T^{\prime \prime}, S\right)$. Hence $f(\mathscr{C})$ is not connected, a contradiction, so the assumption that $x \notin$ int $Q\left(A_{1}\right)$ was false.

We have now established that $Q\left(A_{1}\right)$ is open. So $Q\left(A_{1}\right)$ and $V-Q\left(A_{1}\right)$ are disjoint open-closed subsets of $V$. If $Q\left(A_{1}\right)$ were not connected, there would exist a proper open-closed subset of $Q\left(A_{1}\right)$ (and hence of $V$ ) containing $A_{1}$, which is impossible. It follows that $Q\left(A_{1}\right)$ is an open connected subset of $V$ containing $A_{1}$. Hence, by Theorem $2,2^{X}$ is 1.c. at $A$.

For the converse, suppose that $2^{X}$ is l.c. at each component of $A$. Let $N\left(U_{1}, \cdots, U_{n}\right)$ be a basic open set containing $A$. By Lemma 2 we may assume that $N\left(U_{1}, \cdots, U_{n}\right)$ is essential with respect to $A$. For each component $A_{\alpha}$ of $A$ let $\left\{U_{i_{1}}, \cdots, U_{i_{n_{\alpha}}}\right\}$ be the largest subset of $\left\{U_{1}, \cdots, U_{n}\right\}$ with the property that for each $j=1, \cdots, n_{\alpha}, U_{i_{j}} \cap A_{\alpha} \neq$ $\varnothing$. Then $A_{\alpha} \in N\left(U_{i_{1}}, \cdots, U_{i_{n_{\alpha}}}\right)$. Let $U_{\alpha}=\bigcup_{j=\underline{\alpha}_{1}}^{n_{\alpha}} U_{i_{j}}$. By Theorem 2 there is a connected open set $V_{\alpha}$ such that $A_{\alpha} \subset V_{\alpha} \subset U_{\alpha}$. For each $j=1, \cdots, n_{\alpha}$ let $V_{j}^{\alpha}=V_{\alpha} \cap U_{i_{j}}$. Then

$$
A_{\alpha} \in N\left(V_{1}^{\alpha}, \cdots, V_{n_{\alpha}}^{\alpha}\right) \subset N\left(U_{i_{1}}, \cdots, U_{i_{n_{\alpha}}}\right)
$$

and by Lemma $1, N\left(V_{1}^{a}, \cdots, V_{n_{\alpha}}^{\alpha}\right)$ is connected. Now $A \subset \bigcup_{\alpha}\left(\bigcup_{j \underline{\alpha}_{1}}^{n_{\alpha}} V_{j}^{a}\right)$, and since $A$ is compact, there exist $\alpha_{1}, \cdots, \alpha_{m}$ such that $A \subset$ $\bigcup_{i=1}^{m}\left(\mathbf{U}_{i=1}^{n_{\alpha_{i}}} V_{j}^{\alpha_{i}}\right)$. Then

$$
A \in N\left(V_{1}^{\alpha_{1}}, \cdots, V_{n_{\alpha_{1}}}^{\alpha_{1}}, \cdots, V_{1}^{\alpha_{m}}, \cdots, V_{n_{\alpha_{m}}}^{\alpha_{m}}\right)=\mathscr{C} \subset N\left(U_{1}, \cdots, U_{n}\right) .
$$

The last inclusion follows from the construction and the fact that $N\left(U_{1}, \cdots, U_{n}\right)$ is essential with respect to $A$. 
Let $B, C \in \mathscr{Y} \cap F(X)$ and for and $i=1, \cdots, m$ let $B_{i}=B \cap$ $\left(\bigcup_{j=1}^{n_{\alpha_{i}}} V_{j}^{\alpha_{i}}\right)$ and $C_{i}=C \cap\left(\bigcup_{j=1}^{n_{a_{i}}} V_{j}^{\alpha_{i}}\right)$. Then $B_{i}, C_{i} \in N\left(V_{1}^{\alpha_{i}}, \cdots, V_{n_{\alpha_{i}}}^{\alpha_{i}}\right) \cap$ $F(X)$. As in the proof of Theorem 2, for each $i=1, \cdots, m$ there exists a connected set $\mathscr{L}_{i}$ in $N\left(V_{1}^{\alpha_{i}}, \cdots, V_{n_{\alpha_{i}}}^{\alpha_{i}}\right) \cap F(X)$ which contains $B_{i}$ and $C_{i}$. Define $f_{1}: \mathscr{L}_{1} \rightarrow \mathscr{V} \cap F(X)$ by $F_{1}(D)=D \cup\left(\bigcup_{i=2}^{m} B_{i}\right)$. Since $f_{1}$ is continuous, $f_{1}\left(\mathscr{L}_{1}\right)$ is a connected subset of $\mathscr{V} \cap F(X)$ containing $B$ and $C_{1} \cup\left(\bigcup_{i=2}^{m} B_{i}\right)$. For each $i=2, \cdots, m-1$ define $f_{i}: \mathscr{L}_{i} \rightarrow \mathscr{V} \cap F(X)$ by $f_{i}(D)=\left(\bigcup_{k=1}^{i-1} C_{k}\right) \cup D \cup\left(\bigcup_{k=i+1}^{m} B_{k}\right)$. Then $f_{i}\left(\mathscr{L}_{i}\right)$ is a connected subset of $\mathscr{V} \cap F(X)$ containing $\left(\bigcup_{k=1}^{i-1} C_{i}\right) \cup\left(\bigcup_{k=i}^{m} B_{i}\right)$ and $\left(\bigcup_{k=1}^{i} C_{i}\right) \cup\left(\bigcup_{k=i+1}^{m} B_{i}\right)$. Define $f_{m}: \mathscr{L}_{m} \rightarrow \mathscr{C} \cap F(X)$ by $f_{m}(D)=$ $\left(\bigcup_{i=1}^{m-1} C_{i}\right) \cup D$. Then $f_{m}\left(\mathscr{L}_{m}\right)$ is a connected subset of $\mathscr{V} \cap F(X)$ containing $\left(\bigcup_{i=1}^{m-1} C_{i}\right) \cup B_{m}$ and $C$. Hence $\bigcup_{i=1}^{m} f_{i}\left(\mathscr{L}_{i}\right)$ is a connected subset of $\mathscr{V} \cap F(X)$ containing $B$ and $C$. It follows that $\mathscr{V} \cap F(X)$ is connected, and since $\mathscr{V} \cap F(X)$ is dense in $\mathscr{V}, \mathscr{V}$ is connected. Hence $2^{X}$ is l.c. at $A$.

CoRollary 6. Let $A \in 2^{X}$. If $X$ is c.i.k. (l.c.) at each point of $A$, then $2^{X}$ is c.i.k. (l.c.) at $A$.

The converses of Corollary 6 are false. It is easy to verify (see Lemma 2 of [1]) that for any continuum $X, 2^{X}$ is l.c. at $X$.

COROLLARY 7. The following are equivalent:

(1) For each $i=1, \cdots, n, X$ is c.i.k. (l.c.) at $p_{i}$.

(2) For each $i=1, \cdots, n, 2^{X}$ is c.i.k. (l.c.) at $\left\{p_{i}\right\}$.

(3) $2^{X}$ is c.i.k. (l.c.) at $\left\{p_{1}, \cdots, p_{n}\right\}$.

\section{REFERENCES}

1. J. T. Goodykoontz, Jr., Aposyndetic properties of hyperspaces, Pacific J. Math., 47 (1973), 91-98.

2. J. G. Hocking and G. S. Young, Topology, Addison-Wesley Pub. Co., Reading, Mass., 1961.

3. J. L. Kelley, Hyperspaces of a continuum, Trans. Amer. Math. Soc., 52 (1942), $23-36$.

4. K. Kuratowski, Topology II, Academic Press, New York and London, 1968.

5. E. Michael, Topologies on spaces of subsets, Trans. Amer. Math. Soc., 71 (1951), $152-182$.

6. J. T. Rogers, Jr., The cone equals hyperspace property, Canad. J. Math., 24 (1972), 279-285.

7. M. Wojdyslawski, Retractes absolus et hyperespaces des continus, Fundamenta Mathematicae, 32 (1939), 184-192.

Received April 30, 1973.

WEST VIRGINIA UNIVERSITY 



\section{PACIFIC JOURNAL OF MATHEMATICS}

\section{EDITORS}

RICHARD ARENS (Managing Editor)

University of California

Los Angeles, California 90024

\section{J. DUGUNDJI}

Department of Mathematics University of Southern California Los Angeles, California 90007

D. Gilbarg and J. Milgram

Stanford University

Stanford, California 94305

University of Washington
Seattle, Washington 98105

ASSOCIATE EDITORS
E. F, BECKENBACH
B. H. NEUMANN
F. WOLF
K. Yoshida

\section{SUPPORTING INSTITUTIONS}

\author{
UNIVERSITY OF BRITISH COLUMBIA \\ CALIFORNIA INSTITUTE OF TECHNOLOGY \\ UNIVERSITY OF CALIFORNIA \\ MONTANA STATE UNIVERSITY \\ UNIVERSITY OF NEVADA \\ NEW MEXICO STATE UNIVERSITY \\ OREGON STATE UNIVERSITY \\ UNIVERSITY OF OREGON \\ OSAKA UNIVERSITY
}

\author{
UNIVERSITY OF SOUTHERN CALIFORNIA \\ STANFORD UNIVERSITY \\ UNIVERSITY OF TOKYO \\ UNIVERSITY OF UTAH \\ WASHINGTON STATE UNIVERSITY \\ UNIVERSITY OF WASHINGTON \\ * * * * \\ AMERICAN MATHEMATICAL SOCIETY \\ NAVAL WEAPONS CENTER
}

The Supporting Institutions listed above contribute to the cost of publication of this Journal, but they are not owners or publishers and have no responsibility for its content or policies.

Mathematical papers intended for publication in the Pacific Journal of Mathematics should be in typed form or offset-reproduced, (not dittoed), double spaced with large margins. Underline Greek letters in red, German in green, and script in blue. The first paragraph or two must be capable of being used separately as a synopsis of the entire paper. Items of the bibliography should not be cited there unless absolutely necessary, in which case they must be identified by author and Journal, rather than by item number. Manuscripts, in duplicate if possible, may be sent to any one of the four editors. Please classify according to the scheme of Math. Rev. Index to Vol. 39. All other communications to the editors should be addressed to the managing editor, or Elaine Barth, University of California, Los Angeles, California, 90024.

100 reprints are provided free for each article, only if page charges have been substantially paid. Additional copies may be obtained at cost in multiples of 50 .

The Pacific of Journal Mathematics is issued monthly as of January 1966. Regular subscription rate: $\$ 72.00$ a year (6 Vols., 12 issues). Special rate: $\$ 36.00$ a year to individual members of supporting institutions.

Subscriptions, orders for back numbers, and changes of address should be sent to Pacific Journal of Mathematics, 103 Highland Boulevard, Berkeley, California, 94708.

PUBLISHED BY PACIFIC JOURNAL OF MATHEMATICS, A NON-PROFIT CORPORATION

Printed at Kokusai Bunken Insatsusha (International Academic Printing Co., Ltd.), 270, 3-chome Totsuka-cho, Shinjuku-ku, Tokyo 160, Japan.

Copyright (C) 1973 by Pacific Journal of Mathematics Manufactured and first issued in Japan 


\section{Pacific Journal of Mathematics}

\section{Vol. 53, No. $2 \quad$ April, 1974}

Kenneth Abernethy, On characterizing certain classses of first countable spaces by

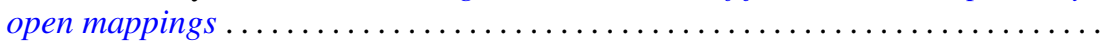

Ross A. Beaumont and Donald Lawver, Strongly semisimple abelian groups .......

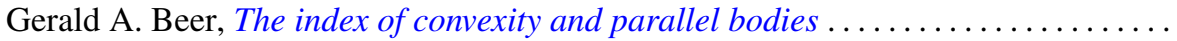

Victor P. Camillo and Kent Ralph Fuller, On Loewy length of rings ..............

Stephen LaVern Campbell, Linear operators for which $T^{*} T$ and $T T^{*}$ commute.

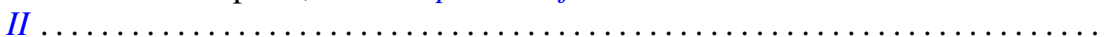

Charles Kam-Tai Chui and Philip Wesley Smith, Characterization of a function by

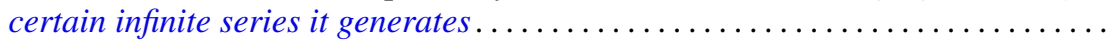

Allan L. Edelson, Conjugations on stably almost complex manifolds . ...........

Patrick John Fleury, Hollow modules and local endomorphism rings . . ..........

Jack Tilden Goodykoontz, Jr., Connectedness im kleinen and local connectedness in

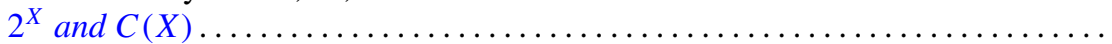

Robert Edward Jamison, II, Functional representation of algebraic intervals .......

Athanassios G. Kartsatos, Nonzero solutions to boundary value problems for

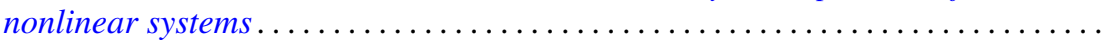

Soon-Kyu Kim, Dennis McGavran and Jingyal Pak, Torus group actions on simply

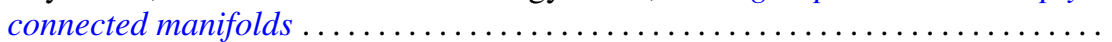

David Anthony Klarner and R. Rado, Arithmetic properties of certain recursively

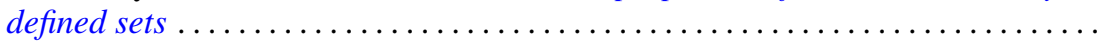

Ray Alden Kunze, On the Frobenius reciprocity theorem for square-integrable

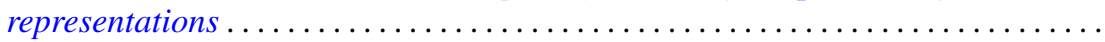

John Lagnese, Existence, uniqueness and limiting behavior of solutions of a class of differential equations in Banach space...

Teck Cheong Lim, A fixed point theorem for families on nonexpansive mappings Lewis Lum, A quasi order characterization of smooth continua

Andy R. Magid, Principal homogeneous spaces and Galois extensions . .

Charles Alan McCarthy, The norm of a certain derivation ..... . .

Louise Elizabeth Moser, On the impossibility of obtaining $S^{2} \times S^{1}$ by elementary surgery along a knot. .

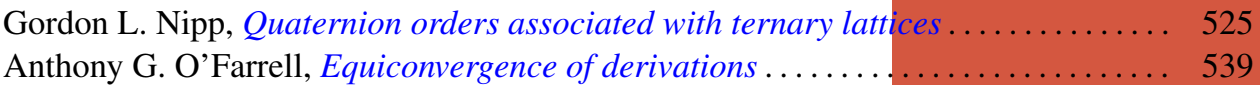

Dorte Olesen, Derivations of $A W^{*}$-algebras are inner . . . . . . . . . . . . . . . 555

Dorte Olesen and Gert Kjærgaard Pedersen, Derivations of $C^{*}$-algebras have

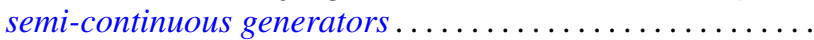

Duane O’Neill, On conjugation cobordism.

Chull Park and S. R. Paranjape, Probabilities of Wiener paths crossing differentiable

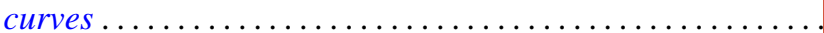

Edward Ralph Rozema, Almost Chebyshev subspaces of $L^{1}(\mu$;

Lesley Millman Sibner and Robert Jules Sibner, A note on the Atiyah-Bott fixed

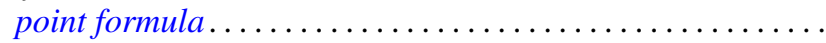

Betty Salzberg Stark, Irreducible subgroups of orthogonal groups generated by

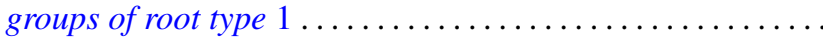

N. Stavrakas, A note on starshaped sets, $(k)$-extreme points and the half ray

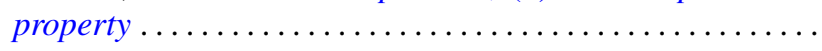

Carl E. Swenson, Direct sum subset decompositions of $Z \ldots \ldots$ 